\title{
Journal of Sustainable Development of Energy, Water and Environment Systems - Volume IV
}

\author{
Neven Duić \\ Editor-in-Chief, Journal of Sustainable Development of Energy, Water and \\ Environment Systems
}

The Journal of Sustainable Development of Energy, Water and Environment Systems - JSDEWES is an international journal dedicated to the improvement and dissemination of knowledge on methods, policies and technologies for increasing the sustainability of development by de-coupling growth from natural resources and replacing them with knowledge based economy, taking into account its economic, environmental and social pillars, as well as methods for assessing and measuring sustainability of development, regarding energy, transport, water, environment and food production systems and their many combinations.

In total 32 manuscripts were published in Volume IV, all of them reviewed by at least two reviewers. The Journal of Sustainable Development of Energy, Water and Environment Systems would like to thank reviewers for their contribution to the quality of the published manuscripts.

1. Prof. Adel Abd El Razek

2. Prof. Bilal Akash

3. Dr. Aleksandra Anić Vučinić

4. Prof. Sabri Avdullahi

5. Prof. Jurij Avsec

6. Ms. Lucie Baborova

7. Dr. Vukman Bakić

8. Prof. Igor Balen

9. Mr. Stefan Balluff

10. Ms. Rosane Battistelle

11. Dr. Cara Beal

12. Prof. Marco Beccali

13. Dr. Susana Boeykens

14. Dr.Stanislav Boldyryev

15. Dr. Heriberto Cabezas

16. Prof. Bin Chen

17. Dr. Milan Cisty

18. Dr. Lidija Čuček

19. Dr. Andras Dallos

20. Prof. Marcello De Falco

21. Prof. Umberto Desideri

22. Ms. Maria Amelia Dias

23. Dr. Ilze Dzene

24. Prof. Göran Finnveden

25. Dr. Alejandro Garcia

26. Ms. Marla Geller

27. Prof. Kunal Ghosh

28. Dr. Dionysios Giannakopoulos

29. Dr. Damien Giurco

30. Dr. Malgorzata Gumienna

31. Prof. Rajiv Gupta

32. Dr. Eduard Hanslík

33. Dr. Mimi Haryani Hassim

34. Ms. Eimear Heaslip

35. Prof. Patrick Hendrick
36. Prof. Liliana Hernández Bello

37. Prof. Ahmad Houri

38. Prof. Jordanka Ivanova

39. Prof. Aleksandar Jovovic

40. Prof. Shinji Kambara

41. Dr. Genku Kayo

42. Dr. Hulya Keskin Citiroglu

43. Dr. Şiir Kilkiş

44. Prof. Branko Klarin

45. Prof. Jiří Jaromír Klemeš

46. Prof. Chun-Han Ko

47. Prof. Branko Kontic

48. Prof. Goran Krajačić

49. Mr. Lev Ktitorov

50. Prof. Tarik Kupusovic

51. Dr. Mohammad Laskar

52. Prof. Hector Malano

53. Prof. Kaan Manisa

54. Prof. Kumudini Marathe

55. Prof. Anca Marina Marinov

56. Prof. Zoran Markov

57. Dr. Isabel Paula Marques

58. Dr. Brian Vad Mathiesen

59. Mr. Greg McNamara

60. Mr. Jan H. Miedema

61. Prof. Irina Mladenoska

62. Prof. Boris N. Kuznetsov

63. Prof. Salvatore Nicosia

64. Dr. Karina Ojeda Delgado

65. Dr. Adina Pacala

66. Dr. Julio Pedraza Garciga

67. Prof. Matilde Pietrafesa

68. Dr. Jose Maria Ponce-Ortega

69. Dr. Meysam Qadrdan

70. Prof. Vijay Raghavan 
71. Prof. Nikola Rajakovic

72. Prof. Rahul V. Ralegaonkar

73. Prof. Temelko Risteski

74. Dr. Francesco Rizzi

75. Prof. Gianfranco Rizzo

76. Dr. Jaya Narayan Sahu

77. Prof. Ichiro Sakata

78. Mr. Hajime Sasaki

79. Dr. Shree Raj Shakya

80. Prof. Ki-Yeol Shin

81. Mr. Peter Smolek

82. Dr. Vladimir Soldo

83. Dr. Maria-Loredana Soran

84. Dr. Pranpreya Sriwannawit

85. Prof. Gordana Stefanovic

86. Prof. Vladimir Stevanovic

87. Dr. Cong Toan Tran

88. Prof. Krzysztof Urbaniec

89. Dr. Petar Varbanov

90. Prof. Manfred Weissenbacher

91. Dr. Kledi Xhaxhiu

92. Dr. Cihan Yildırım

93. Dr. Suzana Yusup

94. Dr. David Zambrana Vasquez

95. Prof. Kalman Ziha

96. Dr. Katarzyna

Zwarycz-Makles 